\title{
Ängste nehmen,
}

\section{Mut machen, motivieren}

\section{Wie Veränderungsprozesse in Organisationen in ethisch sensiblen Bereichen erfolgreich umgesetzt werden können}

\author{
BÄRBEL OTTO UND IRMGARD \\ SCHROLL-DECKER \\ Bärbel Otto ist Leiterin der Senio- \\ renfachstelle-Betreuungsstelle der \\ Stadt Weiden i.d. Opf., Auditorin \\ FQA (Fachstelle Pflege- und Behin- \\ derteneinrichtungen - Qualitäts- \\ entwicklung und Aufsicht). \\ E-Mail baerbel.otto@weiden.de \\ Prof. Dr. Irmgard Schroll-Decker ist \\ Hochschullehrerin für Sozialpäda- \\ gogik mit den Lehrgebieten Sozial- \\ management und Bildungsarbeit an \\ der Hochschule Regensburg. \\ E-Mail Irmgard.Schroll-Decker@hs- \\ regensburg.de
}

\author{
Am Beispiel eines Projektes zur Reduzierung von \\ Fixierungsmaßnahmen in Altenpflegeeinrichtungen \\ kann gezeigt werden, dass die Verbesserung \\ von Lebensqualität auch in schwierigen \\ Lebenslagen durchaus möglich ist.
}

Pflegebedürftige Menschen, die mit Bettgittern, Gurten am Stuhl oder im Bett fixiert werden ... ein Bild, das den Alltag in Pflegeeinrichtungen prägt. Es ist wissenschaftlich bewiesen, dass diese Bewegungseinschränkungen mehr Schaden als Nutzen anrichten. Warum werden in Pflegeeinrichtungen trotzdem Fixierungsmaßnahmen unreflektiert, regelmäßig und routinemäßig angewandt? Ist es möglich, die Handlungsroutinen $\mathrm{zu}$ unterbrechen und in kompetentes pflegefachliches Handeln umzuwandeln?

Diese Fragen nahm ein Projekt der Betreuungsstelle FQA (Fachstelle Pflege- und Behinderteneinrichtungen - Qualitätsentwicklung und Aufsicht [»Heimaufsicht«]) - der Stadt Weiden in der Oberpfalz als Ausgangspunkt für eine Personal- und Organisationsentwicklung. Mittels Informations-, Bildungs- und Beratungsmaßnahmen sollten körpernahe freiheitsentziehende Maßnahmen (FEM) in der Pflege reduziert werden. Dabei ist hervorzuheben, dass es sich im Umgang mit solchen Maßnahmen um eines der sensibelsten ethischen Themen in der Pflege handelt, dessen Relevanz sich daran misst, dass die Grundrechte der Würde des Menschen und der Freiheit des Menschen im Sinne der Bewegungsfreiheit und Selbstbestimmung berührt werden.

\section{Ursachen für Fixierungen}

Expertinnen und Experten bedauern die Unwissenheit der Pflegeverantwortlichen über den Stand der wissenschaftlichen Erkenntnisse (vgl. Meyer u. a. 2009, 31). Die »Leitlinie FEM « (Meyer u. a. 2009) ist eine Zusammenfassung evidenter Studien, die weltweit zum Umgang mit körpernahen freiheitsentziehenden Maßnahmen durchgeführt wurden. Sie bestätigt, dass körpernahe freiheitsentziehende Maßnahmen tägliche unreflektierte Routine in Pflegeeinrichtungen sind.

Als Gründe für die Anwendung von körpernahen freiheitsentziehenden Maßnahmen werden vorrangig die Verhinderung und Minimierung von Stürzen (Sturzprophylaxe) genannt und die Verhaltensregulierung bei herausfordernden Verhaltensweisen. Die Haltungen und Einstellungen der Entscheidungsträger für und wider solche Maßnahmen sind dabei handlungsleitend (vgl. Meyer u. a. 2009, 31, 41 und 49-51) indem sie die Handlungsroutinen aufrechterhalten oder Optionen für Einstellungs- und Verhaltensänderungen ermöglichen. Im pflegewissenschaftlichen Verständnis hat längst ein Paradigmenwechsel stattgefunden, weg vom fürsorgegeleiteten Schutzgedanken hin zum selbstbestimmten Freiheitsgedanken. 


\section{Einflussfaktoren auf die Handlungskompetenz}

An beruflich Handlungs- und Pflegeverantwortliche (z. B. in Leitungsfunktionen) aber auch an Pflegende werden von außen unter Umständen widersprüchliche Erwartungen gestellt, die sie mit den eigenen fachlichen und ethischen Prinzipien abgleichen müssen (vgl. Kirsch/Wassermann 2009, 109 f.). Das berufliche Handeln ist zudem eingebettet in den Rahmen und die Kultur einer Organisation und Institution, die sich dazu mehr oder weniger bedacht zeigt.

Insbesondere drei psychosoziale Spannungsfelder werden häufig aufgeführt, die für eine weit verbreitete Handlungsunsicherheit und Blockaden überlagert (vgl. Meyer u. a. 2009, 14 und 31).

- Rechtfertigungsdruck und Schuldgefühle sind starke Hemmnisse fachkompetenten Handelns. Neben der weit verbreiteten Haftungsangst (vgl. Becker u. a. 2007, 74 f.), fühlen sich Pflegeverantwortliche dem Druck der Angehörigen ausgesetzt, die meist erwarten, dass "nichts passieren« darf (vgl. Schäufele u. a. 2008, 19). Das im Berufsbild übliche hohe Verantwortungsgefühl generiert Schuldgefühle, auch wenn Pflegeverantwortliche keinen Fehler begangen haben und nicht haften. Vermeintlich sichere Routinen auszuführen oder die Verantwortung auf Angehörige zu schieben, nimmt die Angst.

"In der Pflegewissenschaft gibt es

\section{einen Paradigmenwechsel, weg vom}

fürsorgegeleiteten Schutzgedanken hin

\section{zum selbstbestimmten Freiheitsgedanken«}

bei der Umsetzung pflegefachlichen Wissens im Umgang mit körpernahe freiheitsentziehende Maßnahmen verantwortlich sind (vgl. Klie u. a. 2005, 16-17 und Cheung/Yam 2005, 35):

- Institutionelle Zwänge, wie Personalmangel, Zeitdruck und Dokumentationspflicht können dazu führen, dass sich Routinen einschleichen. Der professionelle Anspruch der Pflegeverantwortlichen an eine wertschätzende Beziehungspflege muss Automatismen weichen, wie der unreflektierten Anwendung von körpernahen freiheitsentziehenden Maßnahmen (vgl. Klie/Pfundstein 2004, 124).

- Eine besondere Herausforderung in der Pflege bildet das Spannungsfeld zwischen den Prinzipien der Förderung der Freiheit und Selbstbestimmung einerseits und der Wahrung der körperlichen Unversehrtheit der Betroffenen andererseits (vgl. Schäufele u. a. 2008, 19 f.). Eine pflegefachliche, situationsabhängige Risikoabwägung wird dabei häufig von einem fürsorgegeleiteten Schutzgedanken der Pflegeverantwortlichen
Zur Überwindung eines paternalistischen Pflegeverständnisses und den Aufbau einer reflektierten beruflichethischen Grundhaltung empfehlen Expertinnen und Experten Schulungsprogramme für Pflegekräfte, um den Stand der wissenschaftlichen Erkenntnisse zu etablieren. Als sehr ergiebig haben sich in neuen Studien (vgl. Koczy u. a. 2011, 334-338 und Gulpers 2010 und Huizing u. a. 2009) multifaktorielle Interventionen gezeigt, insbesondere was eine nachhaltige Einstellungs- und Haltungsveränderung anbetrifft.

\section{Projektziel, Projektverlauf, Projektergebnis}

Das Projekt der Stadt Weiden i.d. Opf. (vgl. Otto 2011) setzte von Beginn auf einen Mehrebenen-Ansatz zur Veränderung des organisationellen Umgangs mit körpernahen freiheitsentziehenden Maßnahmen: Es fokussierte die strategische Ebene (vertreten durch die Führungskräfte), die normative Ebene (z. B. Verankerung von Grundsätzen), die Ebene der Pflegefach- und Hilfskräfte und die externen Entscheidungsträger (z. B. Richter, Ärzte, Betreuer). Durch
Informations-, Schulungs- und Beratungsinstrumente sowie fachlichen Austausch sollten die Entscheidungs- und Handlungskompetenz, sowie das Werte- und Verantwortungsbewusstsein von Pflegeverantwortlichen gestärkt werden.

Um dies zu erreichen, wurde ein auf zunächst zwei Jahre angelegtes Projekt zur Reduzierung von körpernahen freiheitsentziehenden Maßnahmen entwickelt und durchgeführt. Alle acht stationären Pflegeeinrichtungen in der Stadt Weiden i.d. Opf. waren am Projekt beteiligt.

In einem Top-down-Ansatz wurden zunächst die Führungskräfte in den Pflegeeinrichtungen im Umgang mit körpernahen freiheitsentziehenden Maßnahmen geschult und beraten. Sie konnten an verschiedenen Veranstaltungen teilnehmen, wie einer Fachtagung, dem Besuch einer Altenpflegeeinrichtung ohne körpernahe freiheitsentziehende Maßnahmen und einem Erfahrungsaustausch. Als Manager des Veränderungsprozesses sind sie verantwortlich für die Pflegephilosophie in der Einrichtung, für Leitbild und Konzeption. Sie leben Werte vor, machen Mut zur Veränderung, nehmen ihre Mitarbeitenden mit ihren Ängsten und Bedürfnissen ernst und stützen dabei, eine berufliche Haltung zu verteidigen.

Nach den Veranstaltungen für Führungskräfte wurden eintägige InHouse-Schulungen für Pflegekräfte nach dem Schulungskonzept »ReduFix Praxis" (www.redufix.de) durchgeführt. Während der gesamten Projektlaufzeit fanden fallbezogene Beratungen vor Ort in den Einrichtungen statt. Ängste nehmen, Mut machen, motivieren, war die Devise.

Um die Wirksamkeit der Interventionen zu dokumentieren, wurde das Projekt mit einer sozialwissenschaftlichen Vorher-nachher-Studie begleitet. Zwischen Juli 2010 und Juli 2011 konnte die Anzahl der körpernahen freiheitsentziehenden Maßnahmen insgesamt um 41,5 Prozent reduziert werden. Das ist darauf zurückzuführen, dass manche Bewohnerinnen und Bewohner nicht mehr fixiert wurden und bei vielen Neuaufnahmen von vorneherein auf solche Maßnahmen verzichtet wurde.

Obwohl nur in der Hälfte der Einrichtungen Schulungen angeboten werden konnten, gelang es in allen Einrich- 
tungen, die Anzahl der körpernahen freiheitsentziehenden Maßnahmen zu reduzieren. Dabei konnte in Einrichtungen, in denen Mitarbeitende geschult wurden, insgesamt auf doppelt so viele körpernahe freiheitsentziehende Maßnahmen, nämlich 56 Prozent, verzichtet werden, als in Einrichtungen, die bis zum Stichtag noch keine Schulungen anbieten konnten.

Führungskräfte in drei Einrichtungen konnten nicht nur die Vermeidung von körpernahen freiheitsentziehenden Maßnahmen, sondern den Verzicht darauf, konzeptionell im Qualitätsmanagement verankern. In diesen Einrichtungen wurde bewiesen, dass eine sichere Pflege ohne körpernahe freiheitsentziehende Maßnahmen möglich ist.

Pflegeverantwortliche haben Handlungssicherheit im Umgang mit körpernahen freiheitsentziehenden Maßnahmen und mit gefährdenden Situationen erlangt. Eine selbstbewusste, fachlich fundierte und gefestigte Argumentation konnte gegenüber Ärzten, Richtern und rechtlichen Vertretern zu einer externen Akzeptanz des Veränderungsprozesses beitragen. Die Kommunikation mit den Ärzten führte beispielsweise zu aussagekräftigen Diagnosen.

\section{Sieben Erkenntnisse aus dem Veränderungsprozess}

Während des Projektverlaufes gab es erhebliche Unterschiede zwischen den Einrichtungen, was die Dauer des Veränderungsprozesses und die Anzahl der Entfixierungen betrifft. Die Abweichungen können zurückgeführt werden auf die Einstellung und Haltung der jeweiligen Pflegedienstleitungen, also der Manager im Veränderungsprozess. Ausschlaggebend war zumeist, ob sie den Mut aufbrachten, sich ihren eigenen Ängsten und ihrem Fürsorgedenken zu stellen und inwieweit sie in der Lage waren, Führungsverantwortung zu übernehmen. Bei der abschließenden Befragung der Führungskräfte in den Einrichtungen zum Projekt konnten folgende Erkenntnisse gezogen werden:

Fundamentaler Widerstand gegen - den Veränderungsprozess wurde nur selten ausgemacht. Die meisten Pflegekräfte fühlten sich durch die Unterstützung der Vorgesetzten in ihren eigenen Einstellungen und Haltungen im Umgang mit körpernahen freiheitsentziehenden Maßnahmen bestärkt.

Als wichtige Mutmacher und Mo-
tivatoren dienten positive Beispie-
le erfolgreicher Entfixierungen.

2. Als wichtiges Argument, auf 3. körpernahe freiheitsentziehende Maßnahmen zu verzichten und kreative alternative Möglichkeiten der Gefahrenabwehr zu entwickeln, gilt der Wegfall der zeitraubenden Dokumentation.

4. Die Arbeitszufriedenheit der Mitarbeiterinnen und Mitarbeiter ist gestiegen. Das beschrieben insbesondere Führungskräfte der Einrichtungen, die auf körpernahe freiheitsentziehende Maßnahmen verzichten.

5. Eine vorrangig wichtige Aufgabe - der Führungskräfte ist es, Angehörige von Anfang an mit einzubeziehen, bereits bei der Heimaufnahme Aufklärungsarbeit zu leisten und das Ziel einer Pflege transparent zu machen, die möglichst frei ist von körpernahen freiheitsentziehenden Maßnahmen.

6 Die positiven Berichte von ande- ren Pflegeverantwortlichen in der Umgebung haben zögerliche Leitungskräfte motiviert und ihnen die Angst vor der Durchführung und vor Unruhe im eigenen Haus genommen.

7. Nicht eingetretene Befürchtungen - über Reaktionen von Angehörigen und ansteigende Stürze sowie die positiven Reaktionen aus dem Umfeld haben die Veränderung stabilisiert. Reaktanzphänomene wie beispielsweise die fehlenden finanziellen Ressourcen für Alternativen zu körpernahen freiheitsentziehenden Maßnahmen spielten keine Rolle mehr.

\section{Resümee}

Auch in ethisch sensiblen Bereichen ist es möglich, Veränderungen von Haltungen und Einstellungen, von Werte- und Verantwortungsbewusstsein zu bewirken.

Die Resultate bestätigen die Stoßrichtung des multifaktoriellen Interventionsansatzes. Seine Stärke liegt darin, dass die Reduzierung von körpernahen freiheitsentziehenden Maßnahmen als
Bestandteil der Organisationskultur einer Einrichtung gesehen wird, in die alle eingebunden und danach auch von allen vertreten werden. Einem Zurückfallen einzelner hinter bereits erreichte Resultate innerhalb einer Einrichtung, aber auch im Vergleich zu den umliegenden, wird dadurch entgegengewirkt.

Eine kontinuierliche Begleitung und Beratung von außen hat sich bewährt. Die multimodale Herangehensweise des Hörens, Sehens, Nachmachens und Erfahrens hat sich hier als veränderungsförderlich erwiesen.

Sensibilisieren, schulen und beraten - Ängste nehmen, Mut machen, motivieren - ist ein erfolgreiches Rezept. Das Projekt zur Reduzierung von Fixierungsmaßnahmen in den Pflegeeinrichtungen in Weiden i.d. Opf. hat das gezeigt. Pflegefachlich gesehen besteht der Hauptgewinn darin, dass eine menschenwürdige Pflege, die sich am Selbstbestimmungs- und Freiheitsgedanken orientiert, entgegen aller Einwände, problemlos in die Praxis übertragen werden kann.

\section{Literatur}

Becker, Clemens u. a. (2007): ReduFix. Alternativen zu Fixierungsmaßnahmen oder: Mit Recht fixiert?. Hannover: Vinzentz Network.

Cheung, Pracy \& Yam, Bernard (2005): Patient autonomy in physical restraint. In Zs.: International Journal of Older People Nursing 14, H. 3a, S. 34-40.

Gulpers, Math u. a. (2010): Belt restraint reduction in nursing homes: design of a quasiexperimental study. In: BMC Geriatrics 11/2010. In: www.biomedcentral.com/1471-2318/10/11. Haut, Antonie u. a. (2011): Leitlinienreport zur Evidenzbasierten Praxisleitlinie. Vermeidung von freiheitseinschränkenden Maßnahmen in der beruflichen Altenpflege. 1. Überarbeitung. In: www.leitlinie-fem.de/downloads/LL-Report_1_Ueberarbeitung_28022011.pdf. Huizing, Anna u. a. (2009): Preventing the use of physical restraints on residents newly admitted to psycho-geriatric nursing home wards: A cluster-randomized trial. In Zs.: International Journal of Nursing Studies $46, \mathrm{H}$. 4, S. 459-469.

Kirsch, Sebastian \& Wassermann, Josef (2009): Eine Initiative zur Reduzierung von Fixierungsmaßnahmen mit verfahrensrechtlichem Ansatz. In Zs.: Betreuungsrechtliche Praxis, H. 3, S. 109-112.

Klie, Thomas \& Pfundstein, Thomas (2004): Münchner Studie: Freiheitsentziehende 


\section{SGB III-Reform bereits berücksichtigt}

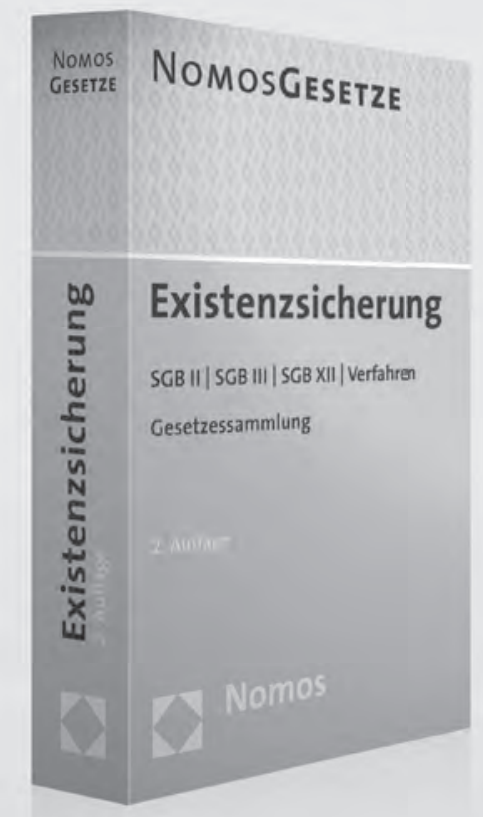

Mit dem Gesetz zur Verbesserung der Eingliederungschancen am Arbeitsmarkt vom 27.12.2011 sind einschneidende Reformen der arbeitsmarktpolitischen Eingliederungsinstrumente beschlossen. Rechtsansprüche sind verändert, Maßnahmen sind gestrichen und das SGB III ist umfassend geändert.

Die Textsammlung berücksichtigt diese umfangreichen Änderungen. Sie bringt neben dem ebenso betroffenen SGB II auch alle anderen (Sozial) Gesetze, die einen notwendigen Bezug zum Existenzsicherungsrecht/Recht der Arbeitsvermittlung aufweisen, auf den allerneuesten Stand. Dies gilt genauso für die relevanten verfahrensrechtlichen Gesetze, die u.a. durch das Gesetz über den Rechtsschutz bei überlangen Gerichtsverfahren Änderungen unterlagen.

Die aktuelle und handliche Ausgabe ist für Berater wie für Betroffene gleichermaßen geeignet.

Weitere Informationen: www.nomos-shop.de/14543

\section{Existenzsicherung}

SGB II | SGB III | SGB XII | Verfahren

Gesetzessammlung 2. Auflage 2012, 799 S., brosch., 9,90€, ISBN 978-3-8329-7346-9

\section{Nomos}

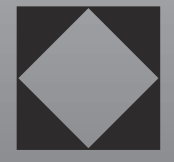

Nomos
Maßnahmen in Münchner Pflegeheimen. In: Hoffmann, Birgit \& Klie, Thomas (2004): Freiheitsentziehende Maßnahmen. Unterbringung und unterbringungsähnliche Maßnahmen in Betreuungsrecht und -praxis. Heidelberg: Müller.

Klie, Thomas \& Pfundstein, Thomas \& Stoffer, Franz Josef (Hg.; 2005): Pflege ohne Gewalt? Freiheitsentziehende Maßnahmen in Pflegeheimen. Entwicklung von Präventions- und Handlungsmaßnahmen. Köln: Kuratorium Deutsche Altershilfe.

Koczy, Petra u. a. (2011): Effectiveness of a Multifactorial Intervention to Reduce Physical Restraints in Nursing Home Residents. In Zs.: Journal of The American Geriatrics Society 59 , S. 333-339.

Meyer, Gabriele u. a. (2009): Leitlinie FEM. Evidenzbasierte Praxisleitlinie. Vermeidung von feiheitseinschränkenden Maßnahmen in der beruflichen Altenpflege. In: www.leitlinie-fem. de/downloads/LeitlinieFEM.pdf.

Otto, Bärbel (2011): Zwischen Fürsorge und Autonomie. Reduzierung körpernaher freiheitsentziehender Maßnahmen in stationären Pflegeeinrichtungen. Ein Projekt der Betreuungsstelle FOA (Fachstelle Pflege- und Behinderteneinrichtungen - Qualitätsentwicklung und Aufsicht) der Stadt Weiden i.d. Opf. Regensburg (unveröff. Masterarbeit).

Schäufele, Martina u. a. (2008): Qualitätsniveau I. Mobilität und Sicherheit bei Menschen mit demenziellen Einschränkungen in stationären Einrichtungen. Heidelberg, München, Landsberg, Berlin: Economica. 
"Versuche nicht, permanent deinen Lebensstandard zu verbessern oder inn gar mit Lebensqualität zu verwechseln." Prof. Dr. Horst Opaschowski, deutscher Erziehungswissenschaftler (geb. 1941)

„Glück stellt sich nicht ein, indem wir es wollen, vielmehr ist Glück die unbeabsichtigte Folge der Wahrnehmung von Verantwortung für Personen und Sachen." Hermann Lübbe, deutscher Philosoph (geb. 1926)

"Wir leben im Zeitalter der Solidarität:

Wir beklagen gemeinsam, was wir einzeln verursachen."

Günter Radtke, deutscher Schriftsteller (geb. 1925)

"Die Absicht, dass der Mensch ıglücklichı sei, ist im Plan der sschöpfungr nicht enthalten." Sigmund Freud, österreichischer Arzt und Psychologe (1856-1939)

"Gesetzt den Fall, Sie hätten einen Manager, der für Ihr Leben verantwortlich ist. Wie würden Sie seine Leistung beurteilen? " Rolf Dobelli, Schweizer Unternehmer und Schriftsteller (geb. 1966)

"Erst hat man kein Glück, und dann kommt auch noch Pech dazu." Jürgen Wegmann, deutscher Fußballspieler (geb. 1964)

„Das Wesen des Guten ist: Leben erhalten, Leben fördern, Leben auf seinen höchsten Wert bringen.

Das Wesen des Bösen ist: Leben vernichten, Leben schädigen, Leben in seiner Entwicklung hemmen.

Das Grundprinzip der Ethik ist also Ehrfurcht vor dem Leben."

Albert Schweitzer, deutsch-französischer Theologe,

Musiker und Arzt (1875-1965) 